\title{
Numeric syntaxonomical analysis of communities of the Molinia caerulea complex in Southwestern of Ukraine
}

\author{
Vasyl V. Budzhak ${ }^{1}$, Illia I. Chorney', Alla I. Tokariuk \& Anna A. Kuzemko,*
}

Key words: management, mowing, Molinia arundinacea, $M$. caerulea, ordination, Prut-Siret interfluve, semi-dry grasslands, syntaxonomy, wet grasslands.

Ključne besede: gospodarjenje, košnja, Molinia arundinacea, M. caerulea, ordinacija, planotasti greben Prut-Siret, polsuha travišča, sintaksonomija, mokrotni travniki.

Received: 7. 12. 2015

Revision received: 6.7. 2016

Accepted: 6. 7. 2016

Co-ordinating Editor:

Jacqueline Loos

\begin{abstract}
The aims of the paper are: i) to establish the coenotic affinity of each species of the Molinia caerulea complex in the southwest of Ukraine, ii) to present the results of a comparative analysis of the syntaxa in which include Molinia, iii) to explain possible reasons for the expansion of Molinia caerulea in the studied area. The study area ranges from the basins of the Upper Prut River to the Upper Siret River in Ukraine. To determine the coenotical affinity of the studied species, 134 relevés were analysed. The data analysis was carried out using the Modified TWINSPAN algorithm provided by the JUICE software. Ecological features of the communities were analyzed by means of a DCA-ordination and phytoindication assessment. It was revealed that in the studied region the species of the Molinia caerulea complex are characterized by a relatively wide coenotic and ecological range and that they can be attributed to different communities of 4 vegetation classes: Molinio-Arrhenatheretea (Polygono bistortae-Trisetion flavescentis, Molinion cearuleae, Arrhenatherion elatioris alliances), Festuco-Brometea (Bromion erecti alliance), Mulgedio-Aconitetea (Calamagrostion villosae alliance), Oxycocco-Sphagnetea (Sphagnion magellanici alliance). This paper presents the results of the floristic composition analyses outlines some ecological peculiarities and discusses the conservational values of the identified syntaxa.
\end{abstract}

\section{Izvleček}

Namen članka je: i) ugotoviti cenološko pripadnost vseh vrst kompleksa Molinia caerulea $\mathrm{v}$ jugozahodni Ukrajini, ii) predstaviti rezultate primerjalne analize sintaksonov z vrstami rodu Molinia, iii) razložiti možne vzroke za razširjanje vrste Molinia caerulea v preučevanem območju. Raziskovano območje se razteza med porečjema zgornjega dela rek Prut in Siret v Ukrajini. Za določitev cenološke pripadnosti preučevanih vrst smo analizirali 134 popisov. Podatke smo analizirali z modificiranim TWINSPAN algoritmom v programu JUICE. Ekološke značilnosti smo analizirali z DCA ordinacijo in fitoindikatorji. Ugotovili smo, da imajo vrste kompleksa Molinia caerulea $\mathrm{v}$ preučevanem območju relativno širok cenotski in ekološki razpon in njihove združbe lahko uvrščamo v štiri razrede: Molinio-Arrhenatheretea (zveze Polygono bistortae-Trisetion flavescentis, Molinion cearuleae, Arrhenatherion elatioris), Festuco-Brometea (zveza Bromion erecti), Mulgedio-Aconitetea (zveza Calamagrostion villosae), Oxycocco-Sphagnetea (zveza Sphagnion magellanici). $\mathrm{V}$ članku predstavljamo rezultate vrstne sestave, izpostavljamo ekološke posebnosti in razpravljamo o naravovarstvenih vrednostih obravnavanih sintaksonov.

1 Yuriy Fedkovych Chernivtsi National University, 11, Fedkovych Str. Chernivtsi, 58012, Ukraine. E-mail: botany@chnu.edu.ua, budzhakv@gmail.com

2 National Dendrological Park “Sofiyivka” NAS of Ukraine, 12a Kyivska Str., Uman, 20300, Ukraine. E-mail: anya_meadow@mail.ru

* Corresponding author 


\section{Introduction}

A decrease in human activity on meadows and pastures in southwestern Ukraine, during the last 20 years, has led to successional changes in grassland communities: Large areas of grasslands have become overgrown with shrub and woody vegetation. This trend has negative consequences for conservation and restoration concerns of meadow communities, like wet and semi-dry Molinia (purple moor-grass) meadows (Prots \& Kagalo 2012) in this region. These meadows form tall, herb- and species-rich communities dominated by Molinia caerulea s.l. and develop under the influence of mowing. Such communities are common in the mountain forest belt and in the foothills ranging from altitudes of $200-1350 \mathrm{~m}$ a.s.l. They can develop under dry and wet soil conditions. In recent years, Molinia meadows have been expanding in the study area. In many cases, the grass species has been observed to change its life strategy and starts to spread rather aggressively to adjacent habitats (Dančak et al. 2012). This trend requires more detailed study.

Molinia is known to expand into neighboring habitats in Western and Central Europe. Particularly, it spreads to ombrotrophic bogs in the Netherlands, which is caused by excessive nitrification (Tomassen et al. 2003). Furthermore, its spread onto heathlands (Friedrich et al. 2011), moorland and subalpine grasslands of Western and Central Europe was observed in connection with changes of habitat management regimes (Chambers et al. 1999, Marrs et al. 2004, Jacquemyn et al. 2005, Hejcman et al. 2010). Molinia caerulea exhibits a high ecological plasticity which allows it to survive in various habitats (Abu Salim et al. 1995, Taylor et al. 2001).

Most of the syntaxonomical reviews declare Molinia caerulea as a diagnostic species of the Molinion alliance, Molinietalia order, Molinio-Arrhenatheretea class (Oberdorfer 1957, Horvat et al. 1974, Mucina et al. 1993, Coldea et al. 1997, Borhidi et al. 2012, Päzolt \& Jansen 2004, Rezníčková 2007, 2014). However, this species shows also relatively high constancies in the syntaxa of other classes, primarily the Scheuchzerio-Caricetea nigrae Tüxen 1937 (syn. Parvo-Caricetea Westhoff in Westhoff et Den Held 1969), Oxycocco-Sphagnetea Br.-Bl. et Tx. ex Westhoff et al. 1946 and Calluno-Ulicetea Br.-Bl. et Tx. ex Klika et Hadač 1944 (incl. Nardetea strictae Oberd. 1949) (Hájek \& Háberová 2001, Šoltés et al. 2001, Berg 2004, Koska \& Timmermann 2004, Krahulec et al. 2007, Hájek \& Hájková 2011, Hájková et al. 2011, Kliment \& Ujházy 2014, Valachovič \& Hegedüšová Vantarová 2014). These communities are characterized by moderately to very wet habitat conditions. However, some authors noted a participation of the Molinia caeru- lea agg. in communities in much drier habitats. They can be assigned to the Molinion alliance as a Molinietum caerulae variant Bromus erectus (Havlova 2006) or Galio veriMolinietum Kącki 2007 (Kącki 2007, 2012). Or, alternatively, to the Bromion erecti alliance of the Brometalia erecti order, in the Festuco-Brometea class as the Brachypodio pinnati-Molinietum arundinaceae association Klika 1939 (Chytrý et al. 2007b, Škodová \& Ujházy 2014). In the latter case, as the name suggests, Molinia caerulea agg. refers to $M$. arundinacea. Thus, it is important to consider the structure of the Molinia caerulea taxonomic complex.

In Central Europe, this complex includes three species that differ in morphology and in the number of chromosomes they carry: Molinia caerulea (L.) Moench, M. arundinacea Schrank subsp. arundinacea and M. arundinacea subsp. freyi (Dančak et al. 2012). They are perennial grasses that form large dense tufts. In Ukraine they are characterized by a broad ecological amplitude ranging from wetlands, wet meadows to forests (Prokudin 1977). The European variant of the Molinia genus is represented by $M$. caerulea, in Ukraine, which is a strong competitor in wet nutrient-poor meadows (Landolt 1977, Taylor et al. 2001, Marrs et al. 2004, Havlova 2006). This competitiveness of the $M$. caerulea can also be explained by the presence of hydrocyanic acid stored in its stems and inflorescences (Tsvelev 1976). Hence, the species is inedible for animals. However, Dančak et al. (2012) suggest a high probability of $M$. arundinacea Schrank subsp. arundinacea Schrank occurring in the Ukraine, as well. According to Tsvelev (1976), the subspecies Molinia caerulea subsp. litoralis (Host) Paul is present in the Carpathians. In contrast to $M$. caerulea; this subspecies grows in open forests, forest clearings, and placers in the lower and middle mountain belts. The author suggests that the priority name of the species is $M$. arundinacea Schrank, although, this is more likely to be $M$. arundinacea Schrank subsp. arundinacea (Schrank) K. Richt.

There are no detailed studies on $M$. caerulea for the study area, yet. But, there is some fragmentary information on the chorology and coenotic affinity of $M$. caerulea in Bukovina and surrounding areas by some researchers (Herbich 1859, Knapp 1872, Berezovskaya 1952, Artemchuk 1954, Shelyag-Sosonko 1960, Horokhova et al. 1974, Yakymchuk 1974, Termena et al. 1992, Tokaryuk et al. 2009).

In particular, Berezovskaya (1952) provides a detailed description of communities with $M$. caerulea, in both low and high or dominant densities in the Mihidra River valley at the area of Vyzhnytsya district of Chernivtsi region. Also, Artemchuk (1954) notes "a significant participation of the M. caerulea" in vegetation cover of the Mihidra 
River valley, an "insignificant participation in the herbage of $M$. caerulea" in the valley of the Siret River and its occurrence in the communities of mountain meadows in vicinity of the Ploska village, Putyla district, Chernivtsi region. Yakymchuk (1974) notes M. caerulea in meadow communities of the Prut River valley within Kosiv and Nadvirna districts of the Ivano-Frankivsk region. Horokhova et al. (1974) indicate that Molinia meadows are distributed in the Valley of Small Siret River (Chernivtsi region). Tokaryuk et al. (2009) made the first attempt to apply the Braun-Blanquet classification of the Molinio-Arrhenatheretea class communities to coenoses with M. caerulea in the Prut-Siret interfluve (Bukovina Pre-Carpathians). In the same area Roleček et al. (2014) characterized the most species-rich communities with $M$. caerulea aggr. and, preliminarily, assigned them to the Brachypodio-Molinietum association. Their similarity to the communities of this association in the White Carpathians is also noted.

A review of the literature reveals that Molinia caerulea complexes in south-western Ukraine include one of the two species $-M$. arundinacea and $M$. caerulea - which differ significantly in their coenotic affinity and their preferred environmental conditions as well as in their distribution patterns and population strategy. Of particular interest is. With special regard to the expansion of Molina caerulea complex types to atypical habitats caused by cli- mate change or anthropogenic influences, the aims of the paper are: i) establishing the coenotic affinity of the species of the Molinia caerulea complexes in the southwest of Ukraine, ii) comparing of syntaxa and their participation and iii) explaining possible reasons for the expansion of the Molinia caerulea s.l. in the study area.

\section{Study area}

The studied area was set in the basins of the Upper Prut River and Upper Siret River within Ukraine (Figure 1).

The Prut River basin covers an area of $27500 \mathrm{~km}^{2}$ of which $9,200 \mathrm{~km}^{2}$ lie on Ukraine territory (Solovei et al. 2009). The studied sites in the Upper Prut river system are located in the mountains, in the foothills and in the plains of the basin up to the border of Ukraine (Kostenyuk 2012). The total area of the Siret River basin is $44,835 \mathrm{~km}^{2}$, with $2,070 \mathrm{~km}^{2}$ in the Ukraine. The upper part of the Siret basin is located in the Eastern Carpathians, near the Pokuttya-Bukovyna Carpathians and the Bukovyna Upland. This amounts to a total extend of $11,270 \mathrm{~km}^{2}$.

Brown soils of low thickness (approx. $30-40 \mathrm{~cm}$ ) form the predominant soil type in the studied area. Below follows a layer of gravel and stones. Generally, the water permeability of the soil is low.

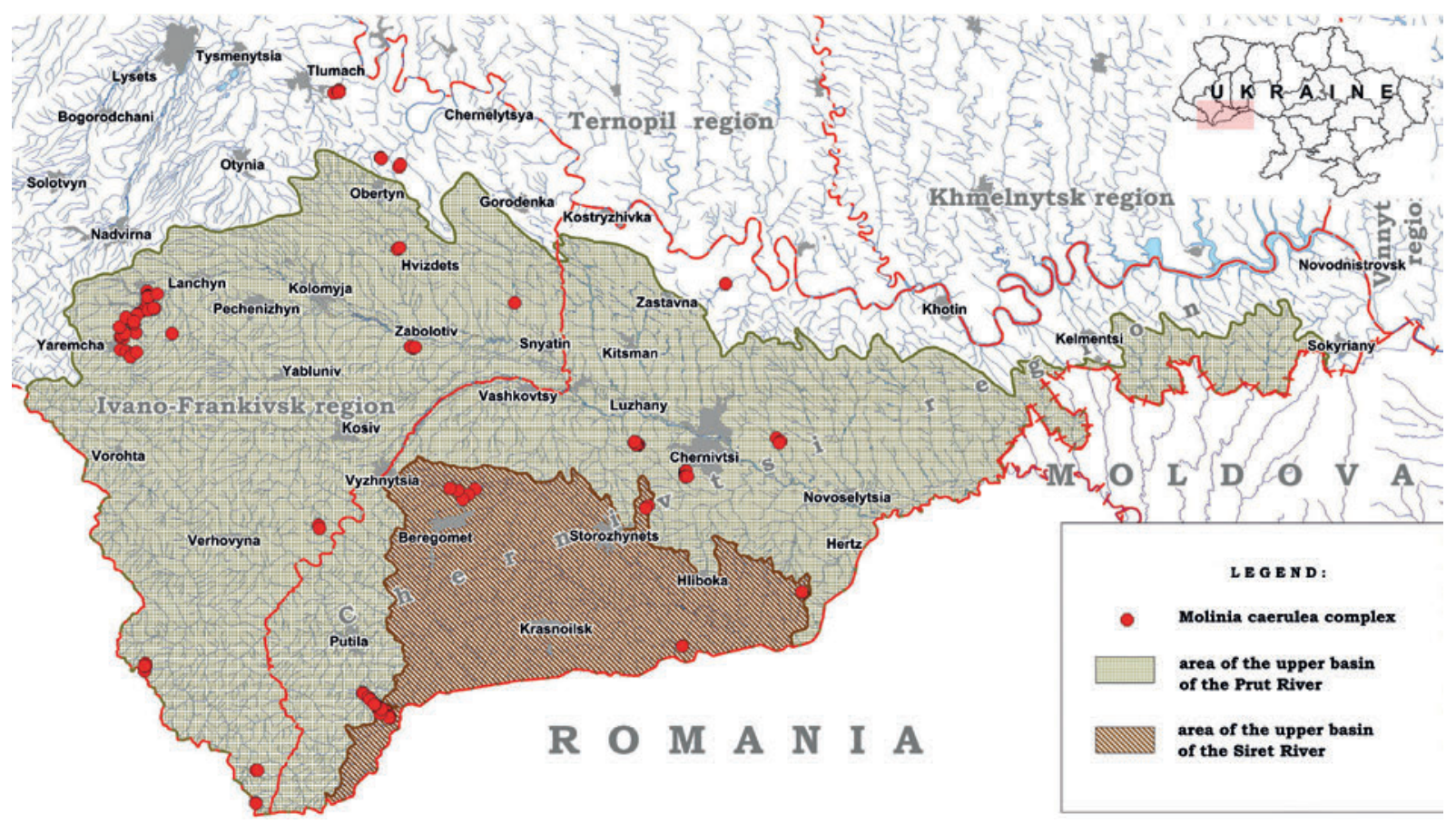

Figure 1: Study area and sites of relevés with participation of the Molinia caerulea complex.

Slika 1: Raziskovano območje in lokacije popisov z vrstami kompleksa Molinia caerulea. 
According to the physical and geographic zoning of Ukraine, the study area is located within the PrutDniester upland of the West-Ukrainian area of deciduous forests of the East European Plain. It includes five physiographic regions (Pre-Carpathian upland, OuterCarpathian, Watershed-Verkhovynska, Polonyna-Chornohora, Marmaroska) of the Ukrainian Carpathians (Marynych et al. 2003). According to the geobotanical zoning of Ukraine, the river basins of Upper Prut and Upper Siret are located in the Maramorosh-ChornohoraSvidovets County characterized by sessile and common oak, beech, larch and spruce forests as well as subalpine and alpine vegetation. Furthermore, they flow through both Opillya-Kremenets County and the Pokuttia-Medobory County (European broadleaved forest region) rich in beech and hornbeam-oak forests, true and steppe meadows as well as meadow steppes. And finally, the river basins are also part of the Bessarabian County with oak and beech forests, steppe meadows and meadow steppes (Eurasian steppe region) (Didukh \& Shelyag-Sosonko 2003). The potentially natural vegetation would be made up offorests like mixed oak-hornbeam forests (F3) or beech and mixed beech forests of both of lowland to submontane types (F5a) and of mountain to alti-mountain types, partially with fir and spruce (F5b) (Bohn et al. 2004).

Climatically, the study area belongs to Ukrainian Carpathians as a sub-region of the Atlantic-Continental region. Atlantic and Mediterranean cyclones cause a significant amount of annual precipitation (in different parts of the sub-region precipitation varies from $600-800$ to $1400-1500 \mathrm{~mm}$ ) depending on local geographical conditions (dissected mountain massifs, their different aspect, slopes and altitude). The average annual temperatures vary from +3 to $+8{ }^{\circ} \mathrm{C}$ (Rudenko 2007). Over the past 50 years the average mean annual temperature of the region has increased by $1.1{ }^{\circ} \mathrm{C}$. Additionally, changes in atmospheric circulation processes, in the recent decades, have led to a redistribution of air masses in the Carpathian region resulting in decreasing precipitation rates in the Pre-Carpathians (Antonov 2002).

\section{Methods}

The data for the present study is compiled of 134 releves surveyed by the authors according to the Braun-Blanquet method, from 2004 to 2015. Additionally, historical relevés from the archive of the Department of Botany, Forestry and Landscape management of the Chernivtsi National University and relevés collected at the territory of the Chernivtsi region of Ukraine were gathered from the literature sources (Berezovskaya 1952, Yakymchuk 1974) (See Figure 1). Although these archived and literary relevés were collected according to the methodology of the Soviet geobotanical school, they were suitable for modern phytosociological studies after minor data refinements. The plot sizes for relevés newly recorded by the authors were either 16 or $25 \mathrm{~m}^{2}$, while the older relevés were $100 \mathrm{~m}^{2}$. Geographic coordinates and the altitude for the new relevés was recorded using GPS navigation (different models) or GoogleEarth software in case of the old relevés. All relevés were entered into the Ukrainian Grassland Database, registered in GIVD as EU-UA-001 (Kuzemko 2012) and the Database of semi-dry grasslands in Ukrainian Pre-Carpathians (EU-UA-002). The relevés are stored in TURBOVEG format (Hennekens $\&$ Schaminee 2001). Nomenclature of plant taxa corresponds to the checklist of Cherepanov (1995).

Analyses were carried out in the programme JUICE (Tichý 2002). The classification was performed using the modified algorithm TWINSPAN (Roleček et al. 2009) with the calculation of the clusters heterogeneity based on the Simpson coefficient. The number of clusters was determined by the algorithm OPTIMCLASS (Tichý et al. 2010) and confirmed by the analysis of the obtained dendrograms.

Diagnostic species of the vegetation units (clusters) were identified with the help of their fidelity value expressed as the phi coefficient (Tichý \& Chytrý 2006). Species with coefficient values above 0.25 were considered to be diagnostic and values above 0.5 as a highly diagnostic. The size of all groups was standardized to equal size. Statistically insignificant values were excluded using Fisher's exact test at $p>0.05$. Identification of the syntaxa was carried out using data of surveys of grassland vegetation from the Ukraine (Kuzemko 2009), as well as from neighboring countries - Romania (Coldea et al. 1997), Hungary (Borhidi et al. 2012), Czech Republic (Chytrý 2007a), Poland (Kącki 2007, 2012), and Slovakia (Hegedüšová Vantarová \& Škodová 2014).

Ordination analysis was performed using the method of DCA-ordination in the R-project software, integrated in the JUICE programme. For the ecological evaluation of the syntaxa, the ecological scales of Didukh (2011) comprised of 12 factors were used (i.e., soil water regime, variability of damping, soil acidity, total salt regime, carbonate content of the soil, nitrogen content of the soil, thermo-regime, humidity of the climate (ombroregime), continentality of the climate, cryo-regime and light). The geographical distribution of plant communities was projected with the help of GIS MapInfo software and the shape file created by the TURBOVEG programme. 
Species were categorised as rare or endangered if they were listed in the International protected lists - Annexes of Bern Convention on the Conservation of European Wildlife and Natural Habitats and Habitat Directive (Council Directive 92/43/EEC of 21 May 1992), current edition of the Red Data Book of Ukraine (Didukh 2009) and in the list of species that are subject to regional protection in the Chernivtsi region (Andrienko \& Peregrym 2012).

\section{Results}

\section{Syntaxonomy}

The classification analysis yielded nine clusters which were identified as associations (Table 1, Electronic Appendix).

Table 1: Synoptic table of the syntaxa with participation of the Molinia caerulea s.l. in south-western Ukraine (numeric values correspond to phi coefficient $\times 100$; only diagnostic species with a value above 0.25 phi coefficient are shown; phi coefficient values for the highly diagnostic species are highlighted in bold).

Tabela 1: Sinoptična tabela sintaksonov z vrsto Molinia caerulea s.l. v jugozahodni Ukrajini (številčne vrednosti predstavljajo fi koeficient $\times 100$; prikazane so samo diagnostične vrednosti s fi koeficientom nad 0,25; fi koeficienti za pomembne diagnostične vrste so prikazani krepko).

Syntaxa/Sintaksoni:

1 - Vaccinio uliginosi-Pinetum mugo

2 - Junco-Molinietum

3 - Sphagno compacti-Molinietum caeruleae

4 - Crepido mollis-Agrostietum capillaris

5 - Anthoxantho odorati-Agrostietum tenuis

6 - Galietum borealis

7 - Com. Molinia arundinacea

8 - Poo-Trisetetum flavescentis

9 - Brachypodio pinnati-Molinietum arundinaceae

\begin{tabular}{|c|c|c|c|c|c|c|c|c|c|}
\hline Syntaxa number & 1 & 2 & 3 & 4 & 5 & 6 & 7 & 8 & 9 \\
\hline Number of relevés & 4 & 8 & 5 & 13 & 41 & 7 & 15 & 14 & 27 \\
\hline Carex pauciflora & 93.5 & --- & --- & -- & --- & --- & -- & --- & -- \\
\hline Pinus mugo & 85.3 & --- & --- & -- & --- & --- & -- & --- & -- \\
\hline Oxycoccus palustris & 85.3 & --- & --- & --- & --- & --- & --- & --- & --- \\
\hline Vaccinium uliginosum & 85.3 & --- & --- & -- & -- & --- & --- & --- & -- \\
\hline Melampyrum saxosum & 85.3 & --- & --- & --- & --- & --- & --- & --- & --- \\
\hline Andromeda polifolia & 68.6 & --- & --- & --- & --- & --- & --- & . & -- \\
\hline Sphagnum cuspidatum & 68.6 & --- & --- & --- & --- & --- & --- & --- & -- \\
\hline Eriophorum vaginatum & 68.6 & -- & --- & -- & -- & --- & -- & --- & -- \\
\hline Eriophorum latifolium & 68.6 & -- & --- & -- & --- & --- & -- & --- & -- \\
\hline Vaccinium vitis-idaea & 52.1 & --- & --- & --- & --- & --- & --- & --- & --- \\
\hline
\end{tabular}

\begin{tabular}{|c|c|c|c|c|c|c|c|c|c|}
\hline Syntaxa number & 1 & 2 & 3 & 4 & 5 & 6 & 7 & 8 & 9 \\
\hline Listera cordata & 47.8 & -- & --- & --- & -- & --- & --- & --- & -- \\
\hline Pleurozium schreberi & 47.8 & -- & --- & -- & -- & --- & -- & --- & -.. \\
\hline Carex flava & --- & 90.5 & --- & --- & --- & --- & --- & --- & --- \\
\hline Parnassia palustris & --- & 84.9 & --- & --- & --- & --- & --- & --- & --- \\
\hline Juncus articulatus & --- & 80.8 & -- & -- & -- & -- & -- & --- & --- \\
\hline Epipactis palustris- & --- & 80.2 & -- & --- & -- & --- & --- & --- & -- \\
\hline Eriophorum polystachyon & --- & 75.5 & --- & --- & --- & --- & --- & --- & -- \\
\hline Crepis paludosa & --- & 72.8 & --- & --- & --- & --- & --- & --- & -- \\
\hline Equisetum palustre & --- & 68.3 & --- & --- & 16 & --- & -- & --- & -- \\
\hline Dactylorhiza majalis & --- & 66.7 & --- & --- & --- & --- & --- & --- & -- \\
\hline Cirsium oleraceum & --- & 55.3 & --- & -- & -- & -- & --- & --- & -- \\
\hline Juncus inflexus & --- & 48.4 & --- & -- & --- & --- & --- & --- & --- \\
\hline Carex paniculata & --- & 47.8 & --- & --- & --- & --- & --- & --- & --- \\
\hline Cirsium palustre & --- & 47.8 & --- & --- & --- & --- & --- & --- & --- \\
\hline Cirsium rivulare & --- & 47.8 & --- & --- & --- & --- & --- & --- & -- \\
\hline Carex nigra & --- & 47.8 & --- & --- & -- & --- & -- & --- & -- \\
\hline Succisa pratensis & --- & 45.1 & -- & --- & 11.4 & --- & --- & 19.1 & -- \\
\hline Juncus effusus & --- & 43 & --- & --- & -- & --- & --- & --- & --- \\
\hline Myosotis palustris & --- & 43 & --- & --- & --- & --- & --- & --- & -- \\
\hline Poa chaixii & --- & $---\varepsilon$ & 88.3 & --- & --- & --- & --- & --- & --- \\
\hline Hypericum alpigenum & --- & --7 & 75.6 & --- & -- & --- & --- & --- & --- \\
\hline Galium hercynicum & --- & ---7 & 75.6 & --- & --- & --- & --- & --- & -- \\
\hline Deschampsia cespitosa & --- & --- & 65.9 & --- & 5.9 & --- & --- & --- & --- \\
\hline Avenella flexuosa & --- & --- & 61.1 & --- & --- & --- & -- & --- & --- \\
\hline Homogyne alpina & --- & --- & 61.1 & --- & --- & --- & --- & --- & 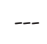 \\
\hline Arnica montana & --- & -- & 58.9 & -- & --- & --- & --- & --- & -- \\
\hline Galium album & --- & -- & 55 & -- & -- & --- & -- & --- & -- \\
\hline Bistorta major & --- & --- & 51.5 & --- & -- & --- & -- & --- & -- \\
\hline Viola declinata & --- & --- & 50.1 & --- & --- & --- & --- & --- & --- \\
\hline Potentilla aurea & --- & --- & 42.6 & --- & --- & --- & --- & --- & --- \\
\hline Festuca ovina & --- & --- & 42.6 & --- & --- & --- & -- & --- & -- \\
\hline Phleum alpinum & --- & --- & 42.6 & --- & -- & --- & --- & --- & -- \\
\hline Achillea carpatica & --- & --- & 42.6 & --- & --- & --- & -- & --- & -- \\
\hline Urtica dioica & --- & --- & 42.6 & -- & -- & --- & -- & --- & -- \\
\hline Luzula sudetica & --- & --- & 37.9 & --- & --- & --- & --- & --- & --- \\
\hline Dianthus compactus & --- & -- & --- & 58.1 & --- & --- & --- & --- & --- \\
\hline Scorzonera rosea & --- & --- & --- & 40.8 & --- & --- & --- & --- & --- \\
\hline Silene nutans & --- & -- & -- & 37.3 & -- & -- & -- & --- & -- \\
\hline Medicago lupulina & --- & --- & --- & 37.3 & --- & --- & --- & --- & --- \\
\hline Stellaria holostea & --- & --- & --- & 33.8 & -- & --- & -- & --- & -- \\
\hline Lotus corniculatus & --- & --- & --- & 28 & 18.2 & --- & --- & --- & --- \\
\hline Rhinanthus alectorolophus & -- & --- & --- & --- & 72.5 & --- & -- & --- & -- \\
\hline Euphrasia vernalis & --- & -- & --- & -- & 69.5 & --- & -- & --- & -- \\
\hline Polygala vulgaris & --- & --- & --- & --- & 68.4 & --- & --- & --- & --- \\
\hline Sisyrinchium septentrionale & e--- & --- & --- & --- & 64.1 & --- & --- & --- & --- \\
\hline Cynosurus cristatus & --- & --- & --- & --- & 55.9 & --- & --- & --- & --- \\
\hline Daucus carota & --- & --- & --- & -- & 51.9 & --- & -- & --- & -- \\
\hline Sieglingia decumbens & --- & --- & --- & --- & 48 & --- & --- & --- & --- \\
\hline Leontodon danubialis & --- & -- & -- & --- & 46.3 & --- & -- & --- & -- \\
\hline Ranunculus acris & --- & -- & --- & --- & 45.2 & --- & --- & --- & --- \\
\hline Gentiana pneumonanthe & --- & --- & --- & --- & 44.7 & --- & -- & --- & --- \\
\hline
\end{tabular}




\begin{tabular}{|c|c|c|c|c|c|c|c|c|c|}
\hline Syntaxa number & 1 & 2 & 3 & 4 & 5 & 6 & 7 & 8 & 9 \\
\hline Thymus roegneri & --- & --- & -- & 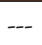 & 44.7 & -- & -- & - & - \\
\hline Leontodon autumnalis & - & - & - & -. & 42.1 & -- & -- & -- & - \\
\hline Senecio jacobaea & --- & --- & --- & --- & 40.2 & --- & --- & --- & -- \\
\hline Nardus stricta & --- & --- & --- & -- & 39.8 & --- & --- & --- & - \\
\hline Ranunculus flammula & -- & -- & -- & -- & 39.3 & -- & -- & --- & 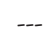 \\
\hline Trifolium pratense & -- & -- & -- & -- & 37.5 & -- & -- & -- & - \\
\hline Agrostis canina & -- & -- & -- & -- & 37 & -- & -- & --- & - \\
\hline Trifolium medium & --- & --- & --- & -- & 36.9 & --- & --- & --- & -- \\
\hline Chrysaspis dubia & -- & --- & -- & -- & 36.4 & --- & -- & -- & - \\
\hline Carex distans & --- & --- & --- & -- & 33.1 & --- & --- & --- & -- \\
\hline Potentilla argentea & -- & -- & -- & -- & 33.1 & -- & --- & -- & - \\
\hline Prunella vulgaris & -- & -- & -- & -- & 32.3 & -- & -- & -- & -- \\
\hline Ononis arvensis & -- & -- & -- & -- & 32.1 & -- & -- & -- & -- \\
\hline Phleum nodosum & -- & -- & -- & -- & 29.6 & -- & -- & -- & -- \\
\hline Leucanthemum vulgare & -- & -- & -- & -- & 29.3 & --- & -- & --- & --- \\
\hline Trifolium repens & --- & --- & --- & --- & 29 & --- & --- & --- & --- \\
\hline Centaurium erythraea & -- & -- & -- & --- & 28 & -- & -- & -- & -- \\
\hline Centaurea jacea & -- & -- & -- & -- & 27 & -- & -- & -- & -- \\
\hline Carex lachenalii & -- & -- & -- & -- & 26.1 & -- & -- & --- & -- \\
\hline Hieracium pilosella & -- & -- & -- & -- & 25.6 & --- & -- & --- & --- \\
\hline Viola tricolor & --- & --- & --- & -- & 25.6 & --- & -- & --- & -- \\
\hline Dactylorhiza maculata & -- & -- & -- & -- & 25.6 & -- & --- & --- & --- \\
\hline Carex aquatilis & -- & -- & -- & -- & 25.6 & -- & -- & -- & -- \\
\hline Chrysaspis aurea & -- & -- & -- & -- & 25.6 & -- & -- & -- & -- \\
\hline Juncus tenuis & -- & --- & --- & --- & 25.6 & -- & -- & -- & -- \\
\hline Juncus conglomeratus & -- & --- & -- & --- & 25.1 & --- & -- & --- & -- \\
\hline Heracleum sphondylium & -- & --- & --- & -- & -- & 77.8 & -- & -- & -- \\
\hline Galium boreale & -- & -- & -- & -- & -- & 55.2 & -- & -- & 21.4 \\
\hline Veratrum album & -- & --- & --- & -- & -- & 52.1 & -- & -- & 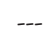 \\
\hline Dianthus stenocalyx & -- & -- & -- & -- & -- & 51.2 & -- & -- & -- \\
\hline Phalacroloma annuum & -- & --- & -- & --- & -- & 49.8 & 18.1 & -- & --- \\
\hline Veronica chamaedrys & -- & -- & -- & 25 & -- & 47.9 & -- & 22 & -- \\
\hline Calamagrostis arundinacea & -- & -- & -- & -- & -- & 47.8 & -- & -- & -- \\
\hline Gladiolus imbricatus & -- & -- & -- & -- & -- & 47.7 & 22.3 & -- & --- \\
\hline Equisetum pratense & -- & -- & -- & -- & -- & 47.5 & -- & -- & --- \\
\hline Valeriana officinalis & -- & -- & -- & -- & -- & 44.1 & -- & -- & --- \\
\hline Geranium sylvaticum & -- & -- & -- & -- & -- & 36.5 & -- & -- & -- \\
\hline Poa pratensis & --- & -- & -- & -- & -- & 36 & --- & -- & -- \\
\hline Arrhenatherum elatius & -- & -- & -- & -- & -- & 27.7 & 24.9 & -- & -- \\
\hline Equisetum telmateia & -- & -- & -- & -- & -- & -- & 68.6 & -- & -- \\
\hline Laserpitium latifolium & -- & --- & -- & -- & -- & -- & 64.4 & --- & --- \\
\hline Dianthus membranaceus & -- & -- & -- & -- & -- & -- & 63.2 & -- & -- \\
\hline Pteridium aquilinum & -- & -- & --- & --- & -- & -- & 60.6 & -- & -- \\
\hline Peucedanum oreoselinum & -- & --- & -- & -- & -- & -- & 57.4 & -- & -- \\
\hline Adenophora lilifolia & -- & -- & -- & -- & -- & -- & 55.5 & -- & -- \\
\hline Convallaria majalis & -- & -- & -- & -- & -- & -- & 49.4 & -- & -- \\
\hline Pedicularis exaltata & -- & -- & -- & -- & -- & -- & 49.4 & -- & -- \\
\hline Ferulago sylvatica & -- & -- & -- & -- & -- & -- & 45.6 & -- & -- \\
\hline Helianthemum ovatum & -- & --- & --- & --- & -- & --- & 42.6 & -- & -- \\
\hline Lathyrus niger & -- & -- & --- & -- & -- & -- & 38.3 & -- & -- \\
\hline Veratrum nigrum & -- & --- & -.- & --- & --. & -- & 37.5 & -- & --. \\
\hline
\end{tabular}

\begin{tabular}{|c|c|c|c|c|c|c|c|c|c|}
\hline Syntaxa number & 1 & 2 & 3 & 4 & 5 & 6 & 7 & 8 & 9 \\
\hline Oberna behen & -- & -- & --- & -- & -- & -- & 34.9 & -- & -- \\
\hline Stellaria media & --- & --- & --- & -- & --- & --- & 34.7 & -- & --- \\
\hline Geranium pratense & --- & --- & --- & --- & --- & --- & 34.7 & -- & --- \\
\hline Iris graminea & -- & --- & --- & --- & -- & --- & 34.7 & -- & --- \\
\hline Thalictrum lucidum & --- & --- & --- & --- & --- & --- & 33.4 & --- & --- \\
\hline Betonica officinalis & --- & --- & --- & --- & -- & --- & 33.2 & -- & -- \\
\hline Campanula persicifolia & --- & --- & --- & --- & --- & --- & 32.8 & -- & --- \\
\hline Trifolium montanum & --- & --- & --- & --- & --- & --- & 30.7 & -- & 24.9 \\
\hline Allium scorodoprasum & --- & --- & --- & -- & -- & --- & 29.7 & -- & --- \\
\hline Campanula rapunculoides & --- & --- & --- & --- & --- & --- & 27.7 & -- & --- \\
\hline Lilium martagon & -- & --- & --- & --- & --- & --- & 27.2 & -- & --- \\
\hline Traunsteinera globosa & -- & --- & --- & -- & -- & -- & --( & 63.2 & --. \\
\hline Helictotrichon praeustum & --- & --- & --- & --- & -- & --- & --- & 52.3 & --- \\
\hline Trollius europaeus & --- & --- & --- & 15.9 & --- & --- & --- & 51.1 & --- \\
\hline Rumex acetosa & -- & --- & --- & --- & -- & --- & --- & 47.6 & --- \\
\hline Pimpinella major & -- & --- & --- & --- & -- & --- & --- & 45.9 & -- \\
\hline Stellaria graminea & --- & --- & --- & --- & -- & --- & --- & 45.5 & -- \\
\hline Trifolium pannonicum & -- & --- & --- & --- & -- & --- & -- & 44 & --- \\
\hline Ranunculus polyanthemos & --- & --- & --- & --- & --- & --- & --- & 42.7 & 18.1 \\
\hline Pimpinella saxifraga & --- & --- & --- & -- & -- & --- & --- & 42.5 & -- \\
\hline Cuscuta europaea & --- & --- & --- & --- & --- & --- & --- & 40.6 & --- \\
\hline Viola montana & -- & --- & --- & --- & -- & --- & --- & 36.7 & --- \\
\hline Polygonatum verticillatum & --- & --- & --- & --- & -- & --- & --- & 36.4 & --- \\
\hline Galeopsis tetrahit & -- & --- & --- & --- & -- & --- & --- & 35.9 & -- \\
\hline Campanula patula & --- & --- & --- & --- 1 & 17.6 & --- & --- & 32.1 & -- \\
\hline Trisetum flavescens & --- & --- & --- & --- & --- & --- & --- & 31.6 & --- \\
\hline Potentilla erecta & --- & --- & -- & 18.7 & -- & --- & 15.4 & 29.8 & -- \\
\hline Euphrasia picta & --- & --- & --- & --- & --- & --- & --- & 27.1 & -- \\
\hline Peucedanum cervaria & -- & -- & --- & --- & --- & --- & --- & --- & 80 \\
\hline Centaurea scabiosa & --- & --- & --- & --- & --- & --- & --- & --- & 67.9 \\
\hline Salvia verticillata & --- & -- & --- & --- & --- & --- & --- & --- & 67.8 \\
\hline Genista tinctoria & --- & -- & -- & --- & --- & --- & --- & --- & 67.7 \\
\hline Asperula cynanchica & -- & --- & -- & --- & --- & --- & --- & --- & 65 \\
\hline Salvia pratensis & --- & -- & -- & --- & -- & --- & --- & --- & 61.6 \\
\hline Cirsium pannonicum & --- & --- & --- & --- & --- & --- & --- & --- & 60.3 \\
\hline Bupleurum falcatum & --- & --- & --- & --- & --- & --- & --- & --- & 59.2 \\
\hline Carex montana & --- & --- & --- & --- & --- & --- & --- & --- & 58.8 \\
\hline Veronica spicata & --- & --- & -- & --- & -- & --- & --- & -- & 58.6 \\
\hline Thesium linophyllon & -- & -- & --- & --- & -- & -- & --- & -- & 57.9 \\
\hline Scabiosa ochroleuca & -- & -- & -- & -- & -- & --- & --- & --- & 53.7 \\
\hline Geranium sanguineum & --- & -- & --- & -- & --- & --- & --- & --- & 53.6 \\
\hline Linum flavum & --- & -- & --- & --- & -- & --- & --- & --- & 52.2 \\
\hline Festuca valesiaca & --- & -- & -- & --- & --- & --- & --- & --- & 48.7 \\
\hline Melampyrum cristatum & -- & -- & -- & --- & -- & --- & --- & --- & 48.7 \\
\hline Securigera varia & --- & -- & -- & --- & -- & --- & --- & --- & 47.9 \\
\hline Prunella grandiflora & --- & -- & -- & --- & -- & --- & --- & --- & 47.9 \\
\hline Inula ensifolia & --- & -- & --- & --- & -- & --- & --- & --- & 46 \\
\hline Asyneuma canescens & --- & --- & -- & --- & --- & --- & --- & --- & 45 \\
\hline Allium oleraceum & -- & -- & -- & --- & -- & --- & --- & --- & 41.2 \\
\hline Onobrychis viciifolia & --- & -- & --- & --- & --- & --- & --- & --- & 41 \\
\hline Seseli libanotis & --- & --- & -- & -- & --- & --- & --- & --- & 41 \\
\hline
\end{tabular}




\begin{tabular}{|c|c|c|c|c|c|c|c|c|c|}
\hline Syntaxa number & 1 & 2 & 3 & 4 & 5 & 6 & 7 & 8 & \\
\hline Inula salicina & -- & -- & -- & -- & -- & --- & --- & --3 & 39.7 \\
\hline Euphorbia klokovii & -- & -- & -- & --- & -- & --- & -- & --3 & 38.1 \\
\hline Euphorbia cyparissias & --- & --- & --- & --- & -- & --- & --- & --- & 38 \\
\hline Euphorbia angulata & -- & --- & --- & --- & -- & --- & --- & --3 & 36.9 \\
\hline Pulsatilla patens & --- & -- & -- & --- & -- & --- & --- & --3 & 36.6 \\
\hline Thalictrum minus & --- & --- & --- & --- & --- & --- & --- & --3 & 36.6 \\
\hline Veronica teucrium & --- & --- & --- & --- & --- & --- & --- & --3 & 36.6 \\
\hline Inula hirta & --- & --- & --- & --- & --- & --- & --- & $--3^{3}$ & 36.6 \\
\hline Elytrigia intermedia & --- & -- & -- & --- & -- & --- & --- & --3 & 33.7 \\
\hline Senecio umbrosus & --- & --- & -- & --- & --- & --- & --- & ---3 & 33.7 \\
\hline Polygala comosa & -- & --- & -- & --- & --- & --- & --- & --3 & 33.1 \\
\hline Medicago falcata & --- & --- & -- & --- & --- & ---2 & 21.3 & $--3^{3}$ & 32.4 \\
\hline Pulmonaria mollis & --- & --- & -- & --- & -- & --- & --- & --3 & 32.2 \\
\hline Lembotropis nigricans & --- & --- & --- & --- & --- & --- & --- & $--3^{3}$ & 31.6 \\
\hline Teucrium chamaedrys & --- & --- & --- & --- & --- & --- & --- & $--3^{3}$ & 31.6 \\
\hline Campanula bononiensis & -- & -- & --- & --- & -- & --- & --- & --3 & 31.6 \\
\hline Adonis vernalis & --- & --- & --- & --- & --- & --- & --- & $--3^{3}$ & 31.6 \\
\hline Carex humilis & --- & --- & --- & --- & --- & --- & --- & $--3^{3}$ & 31.6 \\
\hline Chamaecytisus bloc & -- & --- & --- & --- & -- & --- & --- & $--3^{3}$ & 31.6 \\
\hline Valeriana collina & -- & --- & -- & --- & -- & --- & --- & --3 & 31.6 \\
\hline Equisetum arvense & --- & --- & -- & --- & -- & --- & --- & ---2 & 29.8 \\
\hline Leontodon hispidus & --- & --- & --- & --- & --- & --- & --- & ---2 & 29.7 \\
\hline Stachys recta & -- & --- & --- & --- & --- & --- & --- & --2 & 28.8 \\
\hline Chamaecytisus albus & --- & -- & --- & --- & -- & --- & --- & ---2 & 28.7 \\
\hline Agrimonia eupatoria & --- & --- & --- & --- & --- & --- & -- & ---2 & 28.1 \\
\hline Crepis praemorsa & --- & --- & --- & --- & -- & --- & -- & ---2 & 25.8 \\
\hline Ranunculus auricomus & --- & -- & --- & --- & -- & --- & -- & ---2 & 25.8 \\
\hline Rosa gallica & --- & --- & --- & --- & --- & --- & --- & ---2 & 25.8 \\
\hline Euphorbia virgultosa & --- & --- & --- & --- & --- & --- & -- & ---2 & 25.8 \\
\hline Galium tinctorium & --- & --- & --- & --- & --- & --- & -- & ---2 & 25.8 \\
\hline Gypsophila altissima & --- & --- & --- & --- & -- & --- & --- & ---2 & 25.8 \\
\hline Euphorbia esula & --- & -- & -- & --- & --- & --- & -- & ---2 & 25.8 \\
\hline Hylotelephium maximum & --- & --- & --- & --- & -- & --- & -- & $--2^{2}$ & 25.8 \\
\hline Linaria vulgaris & --- & --- & --- & --- & --- & --- & --- & ---2 & 25.8 \\
\hline Vaccinium myrtillus & 36.8 & -- & 47 & --- & -- & --- & --- & --- & -- \\
\hline Lathyrus pratensis & --- & 48.6 & --- & --- & --- & 35.7 & -- & --- & --- \\
\hline Filipendula ulmaria & --- & 45.1 & --- & --- & --- & 40 & -- & --- & --- \\
\hline Carex pallescens & --- & 32.6 & --- & --- & ---2 & 25.7 & -- & --- & --- \\
\hline Astrantia major & --- & 25.7 & -- & 25 & --- & --- & ---4 & 43.8 & $3--$ \\
\hline Festuca rubra & -- & --- & 48.2 & --5 & 54.8 & -- & --- & -- & --- \\
\hline Campanula serrata & --- & --- & $--\mathrm{C}$ & 61.5 & -- & -- & ---4 & 42.7 & 7 --- \\
\hline Luzula luzuloides & --- & --- & ---5 & 57.9 & -- & --- & ---4 & 40.4 & 4 --- \\
\hline Hieracium umbellatum & --- & --- & --- & 44.2 & --- & --- & --5 & 58.9 & 9 --- \\
\hline Pyrethrum clusii & --- & --- & --- & 42.9 & --- & --- & -- & 39 & -- \\
\hline Carlina acaulis & --- & --- & --- & 42.3 & --- & --- & ---5 & 56.7 & 7 --- \\
\hline Angelica sylvestris & --- & -- & --- & 42.2 & --- & --- & ---4 & 44.8 & $8--$ \\
\hline Anthoxanthum odoratum & --- & --- & --- & 40 & 48 & --- & -- & --- & --- \\
\hline Achillea millefolium & --- & -- & --3 & 32.4 & -- & --- & --3 & 32.8 & $8--$ \\
\hline Trifolium alpestre & -- & -- & --3 & 30.7 & -- & --- & ---3 & 37.2 & $2--$ \\
\hline Centaurea phrygia & --- & --- & --2 & 28.6 & --- & --- & ---3 & 39.1 & 1 --- \\
\hline Hypericum maculatu & --- & --- & --2 & 28.1 & -- & -- & ---3 & 37.1 & $1--$ \\
\hline
\end{tabular}

\begin{tabular}{|c|c|c|c|c|c|c|c|c|c|}
\hline Syntaxa number & 1 & 2 & 3 & 4 & 5 & 6 & 7 & 8 & 9 \\
\hline Phleum pratense & --- & -- & $\ldots$ & 26.4 & -- & -- & --3 & 36.6 & -- \\
\hline Crepis conyzifolia & --- & --- & -- & 25 & --- & -- & --5 & 56.4 & -- \\
\hline Holcus lanatus & --- & --- & --- & ---3 & 32.6 & -- & --2 & 27.4 & -- \\
\hline Sanguisorba officinalis & -- & --- & -- & --- & --- & 57.53 & 31.1 & --1 & 16.4 \\
\hline Dactylis glomerata & --- & --- & -- & --- & --- & 40.33 & 30.91 & 18.7 & --- \\
\hline Lysimachia vulgaris & --- & --- & -- & --- & --- & 333 & 37.1 & --- & -- \\
\hline Potentilla alba & --- & --- & -- & --- & --- & & 51.1 & --2 & 26.5 \\
\hline Anthericum ramosum & --- & --- & -- & --- & --- & --- & 51 & $-\cdots$ & 51 \\
\hline Brachypodium pinnatum & --- & --- & --- & --- & --- & ---4 & 48.6 & --5 & 52.6 \\
\hline Filipendula vulgaris & --- & --- & -- & --- & --- & ---4 & 45.1 & --- & 39 \\
\hline Pyrethrum corymbosum & --- & --- & -- & --- & -- & --- & 33 & -- & 35 \\
\hline Primula elatior & --- & --- & -- & --- & -- & --3 & 30.3 & ---2 & 29.2 \\
\hline Plantago media & --- & --- & -- & --- & --- & ---2 & 29.8 & ---2 & 26.6 \\
\hline Campanula glomerata & --- & --- & -- & --- & --- & --2 & 29.4 & --3 & 35.1 \\
\hline Knautia arvensis & --- & --- & -- & --- & --- & -- & ---4 & 42.83 & 32.6 \\
\hline Serratula tinctoria & --- & --- & --- & --- & ---5 & 52.83 & 31.2 & --2 & 27.5 \\
\hline
\end{tabular}

Cluster 1 - association Vaccinio uliginosi-Pinetum mugo Lutz 1956 (alliance Sphagnion magellanici Kästner et Flößner 1933, order Sphagnetalia medii Kästner et Flößner 1933, class Oxycocco-Sphagnetea Br.-Bl. et Tx. ex Westhoff et al. 1946).

The cluster includes four relevés from the mountain valley (polonyna) Hytanka in the Ivano-Frankivsk region at more than $1500 \mathrm{~m}$ a.s.l. Communities of the association are rare and unique to the region, like bushes with Pinus mugo and Rhododendron hirsutum (Mugo-Rhododendretum hirsuti), for instance. Communities of this association occupy small areas in forest complexes of the Piceion excelsae Pawłowski et al. 1928 alliance, Piceetalia excelsae Pawłowski et al. 1928 order, and Vaccinio-Piceetea Br.-Bl. in Br.-Bl. et al. 1939 class. The Molinia caerulea complex is characterised by $M$. caerulea (L.) Moench s.str. The mean coverage of the Molinia caerulea s.l. amounts to $12.3 \pm 6.4 \%$. The mean number of species of the relevés of Cluster 1 is $9.25 \pm 1.89$ with altogether four rare species (Table 2).

Cluster 2 - Junco-Molinietum R.Tx 1954 (Molinion caeruleae Koch 1926, Molinietalia Koch 1926, MolinioArrhenatheretea Tx. 1937).

The cluster includes eight relevés, which are mostly from the Ivano-Frankivsk and the Chernivtsi region in the mountains at 856 to $1550 \mathrm{~m}$ a.s.l. and partially also from wetlands or often from gentle slopes in the plain of Dzyurkach (at $432 \mathrm{~m}$ a.s.l.). In the lower mountains (Ploska, Pasichna), communities of this association only cover small areas and are surrounded by species-rich grass-forb mountain haylands of the Arrhenatherion elatioris alliance. At the bottom of the subalpine zone (Hnyetyesa mountain), they are surrounded by spruce forests of the Piceion excelsae alliance, Piceetalia excelsae order, 
Vaccinio-Piceetea class and by subalpine tall-herb meadows of the Calamagrostion villosae alliance, Calamagrostietalia villosae order, Mulgedio-Aconitetea class. The Molinia caerulea complex is characterised by M. caerulea s.str. The mean coverage of the Molinia caerulea s.l. amounts to $34.5 \pm 28.5 \%$, and mean number of the species in relevés amounts to $25.25 \pm 3.77$ with eight rare species were revealed (see Table 2).

Cluster 3 - Sphagno compacti-Molinietum caeruleae Wagnerová in Berciková 1976 (Calamagrostion villosae Pawłowski et al. 1928, Calamagrostietalia villosae Pawłowski et al. 1928, Mulgedio-Aconitetea Hadač et Klika in Klika et Hadač 1944).

Cluster includes five relevés recorded in the Chernivtsi region in the mountains at more than $1500 \mathrm{~m}$ a.s.l. Communities of this cluster form mosaic complexes with coenoses of the Homogyno alpinae-Nardetum strictae Mráz 1956 association, Nardo strictae-Agrostion tenuis Sillinger 1933 alliance, Nardetea strictae Rivas Goday in Rivas Goday et Rivas-Mart. 1963 class. The Molinia caerulea complex is characterised by $M$. caerulea s.str. Mean cover with Molinia caerulea s.l. is $1.3 \pm 0.7 \%$ and the mean number of species in the relevés is $14.2 \pm 4.09$ including three rare species (see Table 2).

Cluster 4 - Crepido mollis-Agrostietum capillaris Ružičková 2004 (Polygono bistortae-Trisetion flavescentis Br.-Bl. et R. Tx. ex Marshall 1947, Poo alpinae-Trisetetalia Ellmauer et Mucina 1993, Molinio-Arrhenatheretea Tx. 1937).

This cluster includes 13 relevés surveyed in the mountains (992 to $1101 \mathrm{~m}$ a.s.l.). Relevés of this cluster were close to communities of the Nardo-Agrostion tenuis alliance of the Nardetea strictae class and the Arrhenatherion elatioris alliance of the Molinio-Arrhenatheretea class. The Molinia caerulea complex is characterised by $M$. caerulea s.str. The mean cover of Molinia caerulea s.l. is $37.8 \pm 25.1 \%$ and the mean number of species per relevé is $29.85 \pm 9.90$. Six species were identified as rare (see Table 2).

Cluster 5 - Anthoxantho odorati-Agrostietum tenuis Sillinger 1933 (Arrhenatherion elatioris Luquet 1926, Arrhenatheretalia Pawłowski et al. 1928, Molinio-Arrhenatheretea Tx. 1937).

The cluster includes 41 relevés from 1949 and 1967 which are from literary and archive sources. The data were collected in the Chernivtsi and Ivano-Frankivsk regions in the valleys of the Myhidra and Prut rivers, mainly from hayfields. These grassland complexes are also quite common in the lower mountains, prevalent on slopes of varying steepness and aspects with a wide range of environmental and edaphic conditions. The Molinia caerulea complex is characterised by $M$. caerulea s.str. and M. arundinacea. The mean cover of Molinia caerulea s.l. is $18.9 \pm 20.9 \%$ and the mean number of species per relevé is $32.37 \pm 6.57$. In communities of this association, eleven rare species were found (see Table 2).

Cluster 6 - Galietum borealis Nowińsli 1928 (Molinion caeruleae Koch 1926, Molinietalia Koch 1926, MolinioArrhenatheretea Tx. 1937).

The cluster includes seven relevés, which are mainly from the plain in the Chernivtsi region (at 273-431 m a.s.l.). Only one relevé is from Ivano-Frankivsk region. The found communities are common in river valleys, alongside roads, between forests and at the foot of the mountains. The Molinia caerulea complex is characterised by the $M$. caerulea s.str. and $M$. arundinacea. The mean cover of Molinia caerulea s.l. is $33.9 \pm 24.8 \%$, mean number of species is $35.14 \pm 13.67$. In communities of this association, five rare species were revealed (see Table 2).

Cluster 7 - Com. Molinia arundinacea (Molinion caeruleae Koch 1926, Molinietalia Koch 1926, MolinioArrhenatheretea Tx. 1937).

Cluster 7 includes 15 relevés which are mainly from the Chernivtsi region (only three relevés from the Ivano-Frankivsk region). Most of the relevés are located on the territory of the tract "Dzyurkach" near the city of Chernivtsi. The communities predominantly represent abandoned hayfields on level ground (295-435 m a.s.l). The peculiarity of the floristic composition of communities belonging to this unit did not allow us to find analogues among the previously described associations. Thus, we consider it as community without rank. These unique grass-forb species-rich meadow complexes have developed under the influence of regular mowing and have replaced cut beech and oak forests. As a result of the ongoing decline of the traditional management, however, scrub encroachment increases and the meadows start to be overgrown by trees and shrubs. This explains the presence of many species typical of forestedges forests in the floristic composition of this cluster. The communities border with the meadow communities of the Arrhenatherion elatioris alliance. The Molinia caerulea complex is characterised by the $M$. arundinacea Shrank. The mean cover of Molinia caerulea s.l. is $21.7 \pm 26.6 \%$. The mean number of species is $37.0 \pm 12.2$. In communities of this association, twelve rare species were identified (see Table 2).

Cluster 8 - Poo-Trisetetum flavescentis Knapp ex Oberd. 1957 (Arrhenatherion elatioris Luquet 1926, Arrhenatheretalia Pawłowski et al. 1928, Molinio-Arrhenatheretea Tx. 1937).

The cluster includes 14 relevés recorded in the mountains at altitudes from 900 to $1111 \mathrm{~m}$ a.s.l. These meadow communities with Molinia caerulea were identified and described for the territory of Putyla low mountains in 
the site "Ploska". Here, they are confined mostly to the southeastern and southwestern slopes where they are prevalent in large areas characterized by long-term traditional management (mowing) and fertilization that has allowed a high species richness to develop. The Molinia caerulea complex is characterised by the $M$. arundinacea. The mean cover of Molinia caerulea s.l. is $30.0 \pm 21.0 \%$, and the mean species number is $38.5 \pm 5.98$. In this cluster, six rare species were identified (see Table 2).

Cluster 9 - Brachypodio pinnati-Molinietum arundinaceae Klika 1939 (Bromion erecti Koch 1926, Brometalia erecti Koch 1926, Festuco-Brometea Br.-Bl. et Tx. ex Soó 1947).

This cluster includes 27 relevés in the Chernivtsi and Ivano-Frankivsk regions. All relevés were in the plains (at $167-303 \mathrm{~m}$ a.s.l.) on the predominantly southern slopes. They occupy small areas, surrounded by the meadow communities of the Arrhenatherion elatioris alliance and semi-dry grasslands of the Cirsio-Brachypodion pinnati Hadač et Klika, 1944 alliance of the Festuco-Brometea Br.-Bl. et Tx. ex Soó 1947 class. The Molinia caerulea complex is characterised by the $M$. caerulea s.str. and $M$. arundinacea. The mean cover of Molinia caerulea s.l. is $23.50 \pm 30.30 \%$. The mean species number is $39.15 \pm 13.47$. Altogether, fifteen rare species were identified in this cluster (see Table 2).

\section{Ordination}

The result of the ordination analysis (Figure 2) shows a clear separation of clusters into two groups.

Clusters 1-5 and cluster 8 representing the "wet" type (Figure 3), and clusters 6,7 and 9, which belong to the "dry" type (Figure 4), are projected on the right and left side, respectively, in the ordination diagrams. The syntaxa of the "wet" type are also characterised by high rates of climate humidity (ombroregime) and soil aeration. The syntaxa of the "dry" group are associated with alkaline soil conditions, a high carbonate content in soil, a high salt regime of soil, a high thermal regime and continentality. Clusters $4-8$ are positioned in the central part of the ordination diagram showing that their ecological amplitude are mostly overlapped to different extents. These clusters are assigned to the MolinioArrhenatheretea class. However, those clusters assigned to other classes - 1 (Oxycocco-Sphagnetea), 3 (MulgedioAconithetea), and 9 (Festuco-Brometea) - are considerably distant to each other. There is only a slight overlap of cluster 9 with cluster 7 and, to a lesser extent, with cluster 6 , which is quite logical considering a floristic similarity of the Com. Molinia arundinacea and Brachypodio pinnati-Molinietum arundinaceae associations.

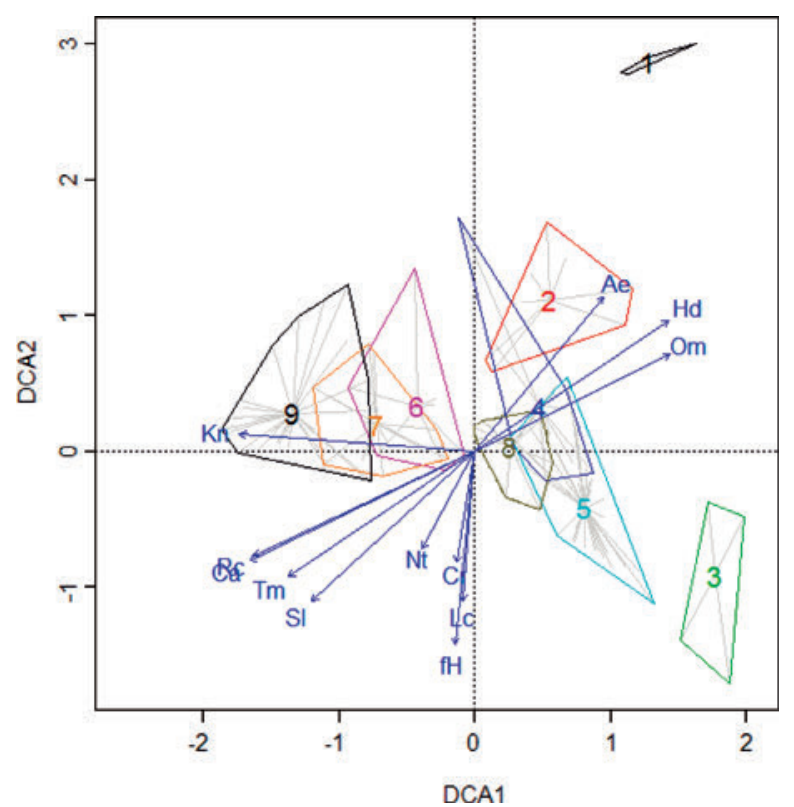

Figure 2: DCA-ordination of the dataset with centroids of the clusters. Number of the centroids corresponds to the numbers of syntaxa in the Table 1. Ecological vectors: Hd - soil water regime, $\mathrm{fH}$ - variability of damping, Rc - soil acidity, Sl - total salt regime, $\mathrm{Ca}$ - carbonate content in soil, $\mathrm{Nt}$ - nitrogen content in soil, Tm thermoregime, $\mathrm{Om}$ - humidity of climate (ombroregime), Kn - continentality of climate, $\mathrm{Cr}$ - cryoregime and Lc - light. Eigenvalues: $1^{\text {st }}$ axis (DCA1) $0.4932,2^{\text {nd }}$ axis (DCA2) 0.2840.

Slika 2: DCA ordinacija s centroidi klastrov. Številka centroida odgovarja številki sintaksona v Tabeli 1 . Ekološki vektorji: Hd - režim vode $\mathrm{v}$ tleh, $\mathrm{fH}$ - variablinost vlažnosti, $\mathrm{Rc}$ - kislost tal, $\mathrm{Sl}$ - skupen režim soli, $\mathrm{Ca}$ - vsebnost karbonata v tleh, $\mathrm{Nt}$ - vsebnost dušika $\mathrm{v}$ tleh, Tm - termorežim (količina sevanja), Om - vlažnost klime (padavinski režim), Kn - kontinentalnost klime, $\mathrm{Cr}$ - kriorežim (temperatura) in Lc - svetloba. Lastne vrednosti: 1 os (DCA1) $0.4932,2$ os (DCA2) 0.2840

\section{Nature conservation values}

Rare and endangered species make up more than 10\% of the species in the studied commmunities and represent about $5 \%$ of the number of vascular plants included in the third edition of the Red Data Book of Ukraine (Didukh 2009) (Table 2). Only one species, Gymnadenia conopsea, characterised by a broad ecological amplitude, occurs in communities of six associations. The other rare species are componen with ts of only one (61\% of species), two (19.5\%) or three (17.1\%) associations.

Thus, communities of mesic and semi-dry grasslands with participation of the Molinia caerulea s.l. are habitats with a high density of rare floristic component in the region. This particulary applies to communities of Anthoxantho odorati-Agrostietum tenuis (cluster 5), Com. Molinia arundinacea (cluster 7) and Brachypodio pinnatiMolinietum arundinaceae (cluster 9). 


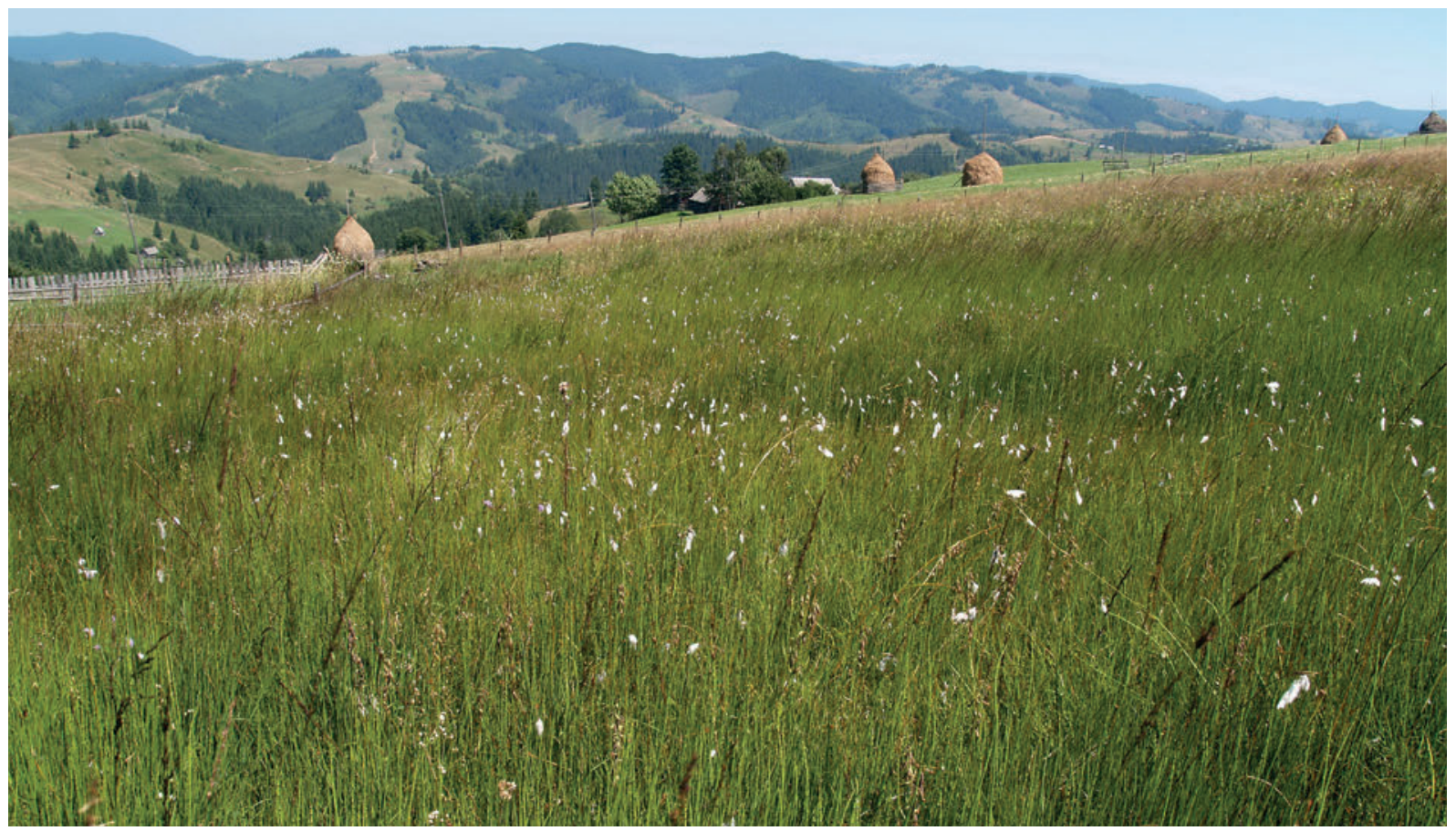

Figure 3: "Wet" type of the purple moor-grass meadows (association Junco-Molinietum) in Huzulska Verkhovyna, a mountain ridge Verkhnia Ploska, Putyla district, Chernivtsi region. Photo: A. I. Tokariuk.

Slika 3: "Vlažni" tip travišč z modro stožko (asociacija Junco-Molinietum) v območju Huzulska Verkhovyna, gorski greben Verkhnia Ploska, okrožje Putyla, regija Chernivtsi. Foto: A. I. Tokariuk.

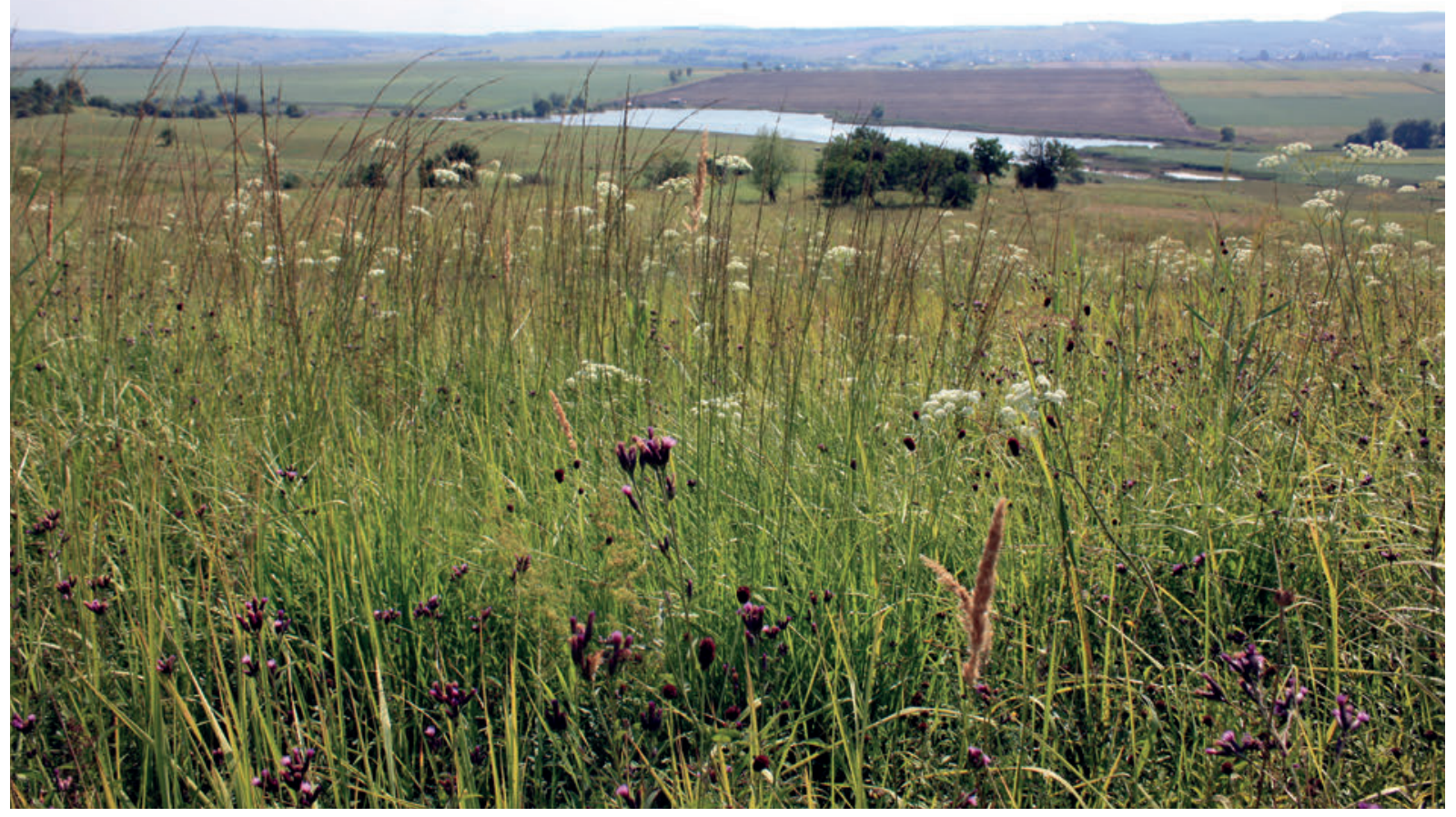

Figure 4: "Dry" type of purple moor-grass meadows (ass. Brachypodio pinnati-Molinietum arundinaceae) in "Maliovanka" site in vicinity of Zavoloka villiage, Storozhynets district, Chernivtsi region. Photo: A.A. Kuzemko.

Slika 4: "Suhi" tip travišč z modro stožko (asociacija Brachypodio pinnati-Molinietum arundinaceae) na območju "Maliovanka" v bližini vasi Zavoloka, okrožje Storozhynets, regija Chernivtsi region. Foto: A.A. Kuzemko. 
Table 2: Frequency of rare and endangered species in syntaxa with participation of $M$. caerulea s.l. in south-west of Ukraine. (Protected status: RDBU - species included in the Red Data Book of Ukraine (2009), RR - regionally rare species, N 2000 species listed in Annexes of the Habitats Directive).

Tabela 2: Frekvenca redkih in ogroženih vrst v sintaksonih z vrsto M. caerulea s. l. v jugozahodni Ukrajini. (Zaščiten status: RDBU - vrste vključene v Rdečo knjigo Ukrajine (2009), RR - regionalno redke vrste, N 2000 - vrste vključene v Dodatek Direktive o habitatih).

\begin{tabular}{|c|c|c|c|c|c|c|c|c|c|c|}
\hline \multirow{2}{*}{ Species name } & \multirow{2}{*}{ Protected status } & \multicolumn{9}{|c|}{ Syntaxa number } \\
\hline & & 1 & 2 & 3 & 4 & 5 & 6 & 7 & 8 & 9 \\
\hline Achillea carpatica & RR & - & - & 20 & - & - & - & - & - & - \\
\hline Aconitum anthora & $\mathrm{RR}$ & - & - & - & - & - & - & - & - & 4 \\
\hline Adenophora lilifolia & N 2000, RR & - & - & - & - & - & - & 33 & - & - \\
\hline Adonis vernalis & RDBU & - & - & - & - & - & - & - & - & 11 \\
\hline Andromeda polifolia & RR & 50 & - & - & - & - & - & - & - & - \\
\hline Arnica montana & N 2000 & - & - & 40 & - & 2 & - & - & - & - \\
\hline Asyneuma canescens & $\mathrm{RR}$ & - & - & - & - & - & - & - & - & 22 \\
\hline Botrychium lunaria & RDBU & - & - & - & 8 & - & - & - & - & - \\
\hline Campanula serrata & N 2000, RR & - & 13 & - & 85 & - & - & - & 64 & - \\
\hline Carex pauciflora & RDBU & 100 & 13 & - & - & - & - & - & - & - \\
\hline Chamaecytisus albus & RDBU & - & - & - & - & - & - & 13 & - & 19 \\
\hline Chamaecytisus blockianus & RDBU & - & - & - & - & - & - & - & - & 11 \\
\hline Clematis integrifolia & $\mathrm{RR}$ & - & - & - & - & - & - & - & - & 4 \\
\hline Colchicum autumnale & RDBU & - & - & - & - & 5 & 14 & 7 & - & - \\
\hline Dactylorhiza cordigera & RDBU & - & 13 & - & - & - & - & - & - & - \\
\hline Dactylorhiza maculata & RDBU & - & - & - & - & 7 & - & - & - & - \\
\hline Dactylorhiza majalis & RDBU & - & 50 & - & - & 2 & - & - & - & - \\
\hline Dianthus compactus & $\mathrm{RR}$ & - & - & - & 54 & - & - & - & 21 & - \\
\hline Dianthus stenocalyx & $\mathrm{RR}$ & - & - & - & - & - & 29 & - & - & - \\
\hline Epipactis palustris & RDBU & - & 88 & - & - & 5 & - & 20 & - & - \\
\hline Galatella linosyris & $\mathrm{RR}$ & - & - & - & - & - & - & - & - & 4 \\
\hline Gentiana pneumonanthe & $\mathrm{RR}$ & - & - & - & - & 22 & - & - & - & - \\
\hline Gladiolus imbricatus & RDBU & - & - & - & - & 22 & 57 & 33 & - & - \\
\hline Gymnadenia conopsea & RDBU & - & 25 & - & 38 & 7 & - & 27 & 43 & 15 \\
\hline Gymnadenia densiflora & RDBU & - & 13 & - & - & - & - & - & - & - \\
\hline Iris hungarica & N 2000, RR & - & - & - & - & - & - & - & - & 4 \\
\hline Iris sibirica & RDBU & - & 13 & - & - & 2 & 14 & - & - & - \\
\hline Leopoldia comosa & RR & - & - & - & - & - & - & 7 & - & - \\
\hline Lilium martagon & $\mathrm{RDBU}$ & - & - & - & 15 & - & - & 20 & - & 4 \\
\hline Melampyrum saxosum & $\mathrm{RR}$ & 50 & - & - & - & - & - & - & - & - \\
\hline Muscari botryoides & $\mathrm{RDBU}$ & - & - & - & - & - & - & - & - & 4 \\
\hline Ophioglossum vulgatum & $\mathrm{RR}$ & - & - & - & - & 2 & - & - & - & - \\
\hline Oxycoccus palustris & $\mathrm{RR}$ & 75 & - & - & - & - & - & - & - & - \\
\hline Pedicularis exaltata & RDBU & - & - & - & - & - & - & 27 & - & - \\
\hline Platanthera bifolia & RDBU & - & - & - & - & 2 & - & - & - & - \\
\hline Pulsatilla grandis & RDBU, BERN & - & - & - & - & - & - & 7 & - & 4 \\
\hline Pulsatilla patens & RDBU, BERN & - & - & - & - & - & - & - & - & 15 \\
\hline Scorzonera purpurea & $\mathrm{RR}$ & - & - & - & - & - & - & - & 7 & 4 \\
\hline Senecio umbrosus & $\mathrm{RR}$ & - & - & - & - & - & - & 7 & - & 19 \\
\hline Traunsteinera globosa & RDBU & - & - & - & - & - & - & - & 43 & - \\
\hline Veratrum nigrum & $\mathrm{RR}$ & - & - & - & - & - & 14 & 27 & - & - \\
\hline \multirow[t]{2}{*}{ Viola declinata } & $\mathrm{RR}$ & - & - & 40 & 8 & - & - & - & 7 & - \\
\hline & & 4 & 8 & 3 & 6 & 11 & 5 & 12 & 6 & 15 \\
\hline
\end{tabular}




\section{Discussion}

Our results showed that Molinia caerulea s.l. has a very broad ecological and coenotic amplitude and occurs in communities of four classes - Molinio-Arrhenatheretea, Mulgedio-Aconitetea, Oxycocco-Sphagnetea and FestucoBrometea, in the south-western part of Ukraine. More often, Molinia is related to hay meadows of the Arrhenatherion elatioris alliance, but not to wet meadows of the Molinion caeruleae alliance, which are typical habitat for Molinia caerulea. The communities of the latter are also present in the studied region, although much rarer.

The results obtained on the expansion of Molinia caerulea s.l. to non-typical habitats confirm the data from Western and Central Europe (Chambers et al. 1999, Jacquemyn et al. 2005, Hejcman et al. 2010 etc.). This development can be explained by the changes of traditional management regimes of the meadows: the Carpathian meadows were traditionally used as hayfields and haymaking served as a regulatory tool to control the distribution of Molinia caerulea. In recent years, haymaking has declined, therein fascilitating the spread of Molinia spread to abandoned hayfields. In the mountains, Molinia communities that grow near to the timber line are quite stable because the altitude prevents shrubs and trees from overgrowing them rapidly. But in the plains, the process of succession occurs more quickly (Bagniuk \& Didukh 2002, Felbaba-Klushyna 2009).

There are interesting changes of the mean coverage of Molinia caerulea in different syntaxa. A low coverage in a non-typical habitat of the Mulgedio-Aconitetea and Oxycocco-Sphagnetea classes of $1 \%$ and $12.3 \%$ respectively is quite plausible. In communities of the MolinioArrhenatheretea class, coverage of Molinia is higher and varies from $18.9 \%$ to $38.7 \%$. The lowest average values of coverage within this class is shown by communities of the Anthoxantho odorati-Agrostietum tenuis, which was identified on the base of historical relevés for the 1949 and 1967. There is evidence that the participation of Molinia caerulea s.l. in meadow phytocenoses was lower in the middle of the last century that it is today because of mowing. The highest average coverage $(38.7 \%)$ was demonstrated by the communities of Crepido mollis-Agrostietum capillaris. This is due to a lack of proper management of the respective sites, which leads to a excessive overgrowth of this species. The mean cover values of the Molinia caerulea s.l. in communities of Bromion erecti are comparable to those of the Molinio-Arrhenatheretea class.

Such prominent role of purple moor-grass in communities of semi-dry grasslands is obviously related to the fact that the Molinia caerulea complex, is characterised by two species - Molinia caerulea (L.) Moench and Molinia arundinacea Schrank (Dančak et al. 2012) in south-west- ern Ukraine and, as in Central Europe. The former grows predominantly in wet and swampy habitats, whereas latter grows in mesic and semi-dry habitats. This is confirmed in this study by the results of the vegetation classification, by structural features of the syntaxa as well as by the results of the ordination. The communities with participation of these species independently demonstrate directed dynamics in the recent decades. In particular, our study revealed the decreasing trend of communities dominated by Molinia caerulea, one the one hand, while, on the other hand, the area of plant communities dominated by $M$. arundinacea is increasing. Factors that affect communities dominated by M. caerulea in the Carpathian Mountains and surrounding areas include the economic development of river valleys, accompanied by destruction of habitats and followed by urban or agricultural development (Bagniuk \& Didukh 2002). In addition, higher temperatures and decreasing precipitation induced by climate change have lead to the drainage of wet habitats and caused a reduction of potential habitats for $M$. caerulae communities. The same factors promote the growth and spread of $M$. arundinacea and its communities which are prevalent in xero-mesic habitats on karst terrain, in dry and semi-dry grasslands habitats. The region of the Transdniestria and Pre-Carpathians as well as low mountains of Ukrainian Carpathians have been subject to the afore mentioned changes (Morgoch 2001, Kobiv 2009). In the foothills of the Carpathians this trend threatens communities in the Dzyurkach and Pidokruh tract, in the vicinity of Chernivtsi, where these unique and extremely species-rich grassland communities, with up to 90 species per $9 \mathrm{~m}^{2}$ occur (Roleček et al. 2014). The floristic composition of these communities harbour a number of rare and endangered species including 14 species of orchids. Molinia arundinacea, however, forms monodominant communities resulting in a loss of biodiversity.

Hence, maintaining the traditional management regime of typical habitats of Molinia caerulea s.l. in the south-western regions of Ukraine should is crucial for their conservation. The management of the communities with participation of Molinia arundinacea and M. caerulea s.str., should be different. To slow down the spread of Molinia arundinacea, mowing should take place in June or July, before seed maturation. For communities with Molinia caerulea s.str., mowing after the maturation of seeds (August-September) will promote the production of sufficient seeds for the diasporas bank to maintain a stable populations of this species. In summary, this suggested regime will help regulate the cover of purple moor-grass and its spread to atypical habitats, on the one hand. On the other hand, it will maintain the high species diversity of these communities including already regionally and nationally endangered species. 


\section{Acknowledgement}

We thank the Orsola Valko and Jacqueline Loos as well as two anonymous reviewers of the manuscript for their valuable comments and suggestions for improvements. Linguistic correction of the English text was made by Roman Volkov and Sioban Loftus.

The study was supported by the Ministry of Education and Science of Ukraine (project 0115U003237).

\section{References}

Abu Salim, K., Gordon, D. B., Shaw, S. \& Smith, C. A. 1995: Variation in Molinia caerulea (L.) Moench, the Purple Moor Grass, in Relation to Edaphic Environments. Annals of Botany 75: 481-489.

Andrienko, T. L. \& Peregrym, M. M. 2012: Official lists of regional rare plants of administrative territories of Ukraine (reference book). Alterpress, Kyyiv, 148 pp [in Ukrainian, with English summary].

Antonov, V. S. 2002: How has the climate of Chernivtsy changed in the last 50 years. Misto, Chernivtsi, 44 pp [in Ukrainian].

Artemchuk, I. V. 1954: Hayfields and pastures of mountain and foothill areas of Chernivtsi region and the ways of their improvement. Scientific notes Chernivtsi State University. Ser. Biological Sciences 4: 34-91 [in Ukrainian].

Bagniuk, V. M. \& Didukh, Y. P. 2002: Ecological problems of Transcarpathia. Proceedings NAUCMA 20: 61-67 [in Ukrainian, with English summary].

Berezovskaya, R. A. 1952: Hayfields and pastures of the Mihodra River valley. Scientific notes Chernivtsi State University. Ser. Biological Sciences 9 (3): 151-199 [in Russian].

Berg, C. 2004: Calluno-Ulicetea - Borstgras-Rasen und trockene Heiden. In: Berg, C., Dengler, J., Abdank, A. \& Isermann, M. (eds.) Die Pflanzengesellschaften Mecklenburg-Vorpommerns und ihre Gefährdung - Textband, Weissdorn, Jena, pp. 290-300.

Bohn, U., Gollub, G., Hettwer, C., Neuhäuslová, Z., Raus, T., Schlüter, H., Weber, H. \& Hennekens, S. (eds.). 2004: Map of the natural vegetation of Europe. Scale $1: 2500$ 000. Interactive CDROM: explanatory text, legend, maps. Bundesamt für Naturschutz, Bonn: CD-ROM + 19 pp.

Borhidi, A., Kevey, B. \& Lendvai, G. 2012: Plant communities of Hungary. Akadémiai Kiadó, Budapest, 544 pp.

Chambers, F. M., Mauquoy, D. \& Todd, P. A. 1999: Recent rise to dominance of Molinia caerulea in environmentally sensitive areas: new perspectives from palaeoecological data. Journal of Applied Ecology 36: 719-733.

Cherepanov, S. K. 1995: Vascular plants of Russia and adjacent states. Mir i Semia, St. Petersburg, 992 p. [in Russian].

Chytrý, M. (ed.) 2007a: Vegetace České republiky. 1. Travinná a keř́čková vegetace. Academia, Praha, 528 pp.
Chytrý, M. 2007b: Festuco-Brometea Br.-Bl. et Tüxen ex Soó 1947. In: Chytrý, M. (ed.), Vegetace České republiky. 1. Travinná a keříčková vegetace. Academia, Praha, pp. 447-449.

Coldea, G., Sanda, V., Popescu, A. \& Ştefan, N. 1997: Les associations végétales de Roumanie. Tome 1 . Les associations herbacées naturelles. Presses Universitaires de Cluj Cluj-Napoca, 261 pp.

Council Directive 92/43/EEC of 21 May 1992 on the conservation of natural habitats and of wild fauna and flora - OJ L 206, 22.7.1992.

Dančak, M., Duchoslav, M. \& Travniček, B. 2012: Taxonomy and cytogeography of the Molinia caerulea complex in central Europe. Preslia 84: 351-374.

Didukh, Ya. P. \& Shelyag-Sosonko, Yu. R. 2003: Geobotanic zoning of Ukraine and adjacent areas. Ukrainian Botanical Journal 60 (1): 6-17. [in Ukrainian with English summary].

Didukh, Ya. P. (ed.) 2009: Red Data Book of Ukraine. Plant kingdom. Kyyiv, Globalkonsalting: 900 pp. [in Ukrainian].

Didukh, Ya. P. 2011: The ecological scales for the species of Ukrainian flora and their use in synphytoindication. Phytosociocentre, Kyyiv, $176 \mathrm{pp}$.

Felbaba-Klushyna, L. M. 2009: Current status, trends and ways of conservation and restoration of biodiversity of plant cover in Transcarpathian Lowland. Scientific Herald of the Uzhgorod University 25: 71-88. [in Ukrainian with English summary].

Friedrich, U., von Oheimb, G, Dziedek, C, Kriebitzsch, W. U, Selbmann, K, \& Härdtle, W. 2011: Mechanisms of purple moorgrass (Molinia caerulea) encroachment in dry heathland ecosystems with chronic nitrogen inputs. Environmental Pollution 159 (12): 3553-3559.

Hájek, M. \& Háberová, I. 2001: Scheuchzerio-Caricetea fuscae. In: Valachovič, M. (ed.) Rastlinné spoločenstvá Slovenska. 3. Vegetácia mokradí. Veda, Bratislava, pp. 185-273.

Hájek, M. \& Hájková, P. 2011: Scheuchzerio palustris-Caricetea nigrae Tüxen 1937. In: Chytrý M. (ed.), Vegetace České republiky. 3. Vodní a mokřadní vegetace. Academia, Praha, pp. 615-619.

Hájková, P., Navrátilová, J. \& Hájek, M. 2011: OxycoccoSphagnetea Br.-Bl. et Tüxen ex Westhoff et al. 1946. In: Chytrý, M. (ed.), Vegetace České republiky. 3. Vodní a mokřadní vegetace. Academia, Praha, pp. 705-708.

Havlova, M. 2006: Syntaxonomical revision of the Molinion meadows in the Czech Republic. Preslia 78: 87-101.

Hegedüšová Vantarová, K. \& Škodová, I. (eds.), 2014: Rastlinné spoločenstvá Slovenska. 5. Travinno-bylinná vegetácia. Veda, Bratislava, $581 \mathrm{pp}$.

Hejcman, M., Češková, M. \& Pavlů, V. 2010: Control of Molinia caerulea by cutting management on sub-alpine grassland. Flora 205: $577-582$.

Hennekens, S. M. \& Schaminee, J. H. J. 2001: TURBOVEG, a comprehensive database management system for vegetation data. Journal of Vegetation Science 12: 589-591. 
Herbich, F. 1859: Flora der Bukowina. Leipzig, 460 pp.

Horokhova, Z. N., Kostevych, Z. K., Solodkova, T. I. \& Strelchenko, T. I. 1974: Flora of the Soviet Bukovina and its rational use. Problems of modern natural science. Proceedings prepared for the 100th anniversary of Chernivtsi State University. Vyshcha shkola, Lviv, pp. 137-143 [in Ukrainain].

Horvat, I., Glavač, V. \& Ellenberg, H. 1974: Vegetation of Südosteuropas. VEB Gustav Fischer Verlag, 768 pp.

Jacquemyn, H., Brys, R., \& Neubert, M. G. 2005: Fire increases invasive spread of Molinia caerulea mainly through changes in demographic parameters. Ecological Applications 15(6): 2097-2108.

Kącki, Z. 2012: Variability and long-term changes in the species composition of Molinia meadows in Poland: a case study using a large data set from the Polish Vegetation Database. Acta Botanica Silesiaca, Monographiae 7: 5-144.

Kącki, Z.: 2007. Comprehensive syntaxonomy of Molinion meadows in southwestern Poland. Acta Botanica Silesiaca, Monographiae 2: 134 pp.

Kliment, J. \& Ujházy, K. 2014: Nardetea strictae. In: Hegedüšová Vantarová, K.\& Škodová, I. (eds.) Rastlinné spoločenstvá Slovenska. 5. Travinno-bylinná vegetácia. Veda, Bratislava, pp. 385-444.

Knapp, J.-A. 1872: Die bischer bekannten Pflanzen Galiciens und der Bukowina. Wilhelm Braumüller, Wien, 520 pp.

Kobiv, Yu. Yo. 2009: Global climate change as a threat to the species biodiversity in the high-mountain zone of the Ukrainian Carpathians. Ukrainian Botanical Journal 66 (4): 451-465 [in Ukrainian with English summary].

Koska, I. \& Timmermann, T. 2004: Parvo-Caricetea - Röhrichte, Großseggenriede und Feuchtstaudenfluren nährstoffreicher Standorte. In: Berg, C., Dengler, J., Abdank, A. \& Isermann, M. (eds.) Die Pflanzengesellschaften Mecklenburg-Vorpommerns und ihre Gefährdung - Textband, Weissdorn, Jena, pp. 163-195.

Kostenyuk, L. V. 2012: Regularities channel formation in the Upper Prut river system. Abstract of the $\mathrm{PhD}$ thesis in Geography. Chernivtsi, 20 pp. [in Ukrainian].

Krahulec, F., Chytrý, M. \& Härtel, H. 2007: Calluno-Ulicetea Br.-Bl. et Tüxen ex Klika et Hadač 1944. In: Chytrý M. (ed.). Vegetace České republiky. 1. Travinná a keříčková vegetace. Academia, Praha, pp. 281-319.

Kuzemko, A. 2012: Ukrainian Grasslands Database. Biodiversity \& Ecology 4: 430-430.

Kuzemko, A. A. 2009: Vegetation of Ukraine. Meadow Vegetation. Molinio-Arrhenatheretea class. Phytosociocentre, Kyyiv, 376 pp. [in Ukrainian with English summary].

Landolt, E. 1977: The importance of closely related taxa for the delimitation of phytosociological units. Vegetatio 34: 179-189.

Marrs, R. H., Phillips, J. D. P., Todd, P. A., Ghorbani, J. \& le Duc, M. G. 2004: Control of Molinia caerulea on upland moors. Journal of Applied Ecology 41: 398-411.

Marynych, O. M., Parkhomenko, H. O., Petrenko, O. M. \& Shyshchenko, P. H. 2003: Improved the physical and geographic zoning Ukraine. Ukrainian Geographical Journal 1: 16-20 [in Ukrainian with English summary].

Morgoch, O. V. 2001: Meso-climatic features of the Ukrainian Carpathians. Scientific notes of Vinnytsya State Pedagogical University named after Michailo Kotzubynsky 1: 27-33 [in Ukrainian with English summary].

Mucina, L., Grabherr, G., Ellmauer, T. (eds.). 1993: Die Pflanzengesellschaften Österreichs. Teil I. Anthropogene Vegetation. Gustav Fisher Verlag, Jena-Stuttgart-New York, 578 pp.

Oberdorfer, E. 1957: Pflanzensoziologie. Süddeutsche Pflanzengesellschaften. Jena 10, 564 pp.

Päzolt, J. \& Jansen, F. 2004. Klasse: Molinio-Arrhenatheretea Tx. 1937 - Wirtschaftsgrünland. In: Berg, C., Dengler, J., Abdank, A. \& Isermann, M. (eds.) Die Pflanzengesellschaften MecklenburgVorpommerns und ihre Gefährdung - Textband, Weissdorn, Jena, pp. 327-335.

Prokudin, Yu. V. (ed.) 1977: Grasses of Ukraine. Naukova Dumka, Kyiv, 518 pp. [in Ukrainain].

Prots, B. \& Kagalo, O. (eds.) 2012: Catalogue of habitat types of the Ukrainian Carpathians and Transcarpathian Lowland. Merkator, Lviv, 294 p. [In Ukrainian].

Řezníčková, M. 2007: Molinion caeruleae Koch 1926. In: Chytrý M. (ed.), Vegetace České republiky. 1. Travinná a keříčková. Academia, Praha, pp. 209-210.

Řezníčková, M. 2014: Molinion caeruleae. In: Hegedüšová Vantarová, K., Škodová, I. (eds.), 2014: Rastlinné spoločenstvá Slovenska. 5. Travinno-bylinná vegetácia. Veda, Bratislava, pp. 305-314.

Roleček, J., Čornej, I.I. \& Tokarjuk, A.I. 2014: Understanding the extreme species richness of semi-dry grasslands in east-central Europe: a comparative approach. Preslia 86: 13-34.

Roleček, J., Tichý, L., Zelený, D. \& Chytrý, M. 2009: Modified TWINSPAN classification in which the hierarchy respects cluster heterogeneity. Journal of Vegetation Science 20: 596-602.

Rudenko, L.G. (ed.) 2007: National Atlas of Ukraine. DNVP "Kartografiya", Kyyiv. 435 pp. [in Ukrainian.].

Shelyag-Sosonko, Yu. R. 1960: The main types of grasslands in valleys of the Dniester River in borders of the western regions of the UkrSSR. Scientific Yearbook. Department of Biology. Chernivtsi, pp. 402-407 [in Russian].

Škodová, I. \& Ujházy, K. 2014: Bromion erecti . In: Hegedüšová Vantarová, K., Škodová, I. (eds.). Rastlinné spoločenstvá Slovenska. 5. Travinno-bylinná vegetácia. Veda, Bratislava, pp. 117-130.

Solovei, T., Hrushchynskyi, T. \& Yuzviak, K. 2009: Atlas of surface water of the Prut basin (in Ukraine). PP Moshynskyi V.S., KanianetsPodilskyi, 21 pp. [in Ukrainian].

Šoltés, R., Hájek, M. \& Valachovič, M. 2001: In: Valachovič, M. (ed.) Rastlinné spoločenstvá Slovenska. 3. Vegetácia mokradí. Veda, Bratislava, pp. 275-296.

Taylor, K., Rowland, P. \& Jones, H.E. 2001: Molinia caerulea (L.) Moench. Journal of Ecology 89: 126-144. 
Termena, B.K., Stefanyk, V.I., Serpokrylova, L.S., Yakymchuk, M.K., Bazhanova, N.V., Vainahiy, V.I., Smolinska, M.O. \& Chorney, I.I. 1992: Synopsis of flora of the Northern Bukovina (vascular plants). Publishing of Newspaper "From Dniester to Carpathians", Chernivtsi, 226 pp. [in Ukrainian].

Tichý, L. 2002: JUICE, software for vegetation classification. Journal of Vegetation Science 13: 451-453.

Tichý, L. \& Chytrý, M. 2006: Statistical determination of diagnostic species for site groups of unequal size. Journal of Vegetation Science 17: 809-818.

Tichý, L., Chytrý, M., Hájek M., Talbot, S. S. \& Botta-Dukát, Z. 2010: OptimClass: Using species-to-cluster fidelity to determine the optimal partition in classification of ecological communities. Journal of Vegetation Science 21 (2): 287-299.

Tokaryuk, A. I., Korotchenko, I. A. \& Budzhak, V. V. 2009: MolinioArrehenatheretea communities with the participation of rare species in Pruth-Sireth Interfluent (Bukovynske Prykarpattya). Nature reserves in Ukraine 15 (1): 7-22 [in Ukrainian with English summary].
Tomassen, H. B. M., Smolders, A. J. P., Lamers, L. P. M. \& Roelofs, J. G. M. 2003: Stimulated growth of Betula pubescens and Molinia caerulea on ombrotrophic bogs: role of high levels of atmospheric nitrogen deposition. Journal of Ecology 91: 357-370.

Tsvelev, N. N. 1976: Grasses of the URRS. Nauka, Leningrad, pp. 556-558 [in Russian].

Valachovič, M. \& Hegedüšová Vantarová, K. 2014: Calluno-Ulicetea In: Hegedüšová Vantarová, K. \& Škodová, I. (eds.) 2014: Rastlinné spoločenstvá Slovenska. 5. Travinno-bylinná vegetácia. Veda, Bratislava, pp. 447-462.

Yakymchuk, M. K.1974: Meadow of Pokuttya Pre-Carpathians and ways of their improvement. Ukrainian Botanical Journal 31 (5): 605-609 [in Ukrainian].

\section{Electronic Appendix:}

www.degruyter.com/view/j/hacq

ojs.zrc-sazu.si/hacquetia

Appendix Table 1: Phytocoenotic table of the syntaxa with participation of the Molinia caerulea s.l. in southwestern of Ukraine (clusters 1-5).

Appendix Table 2: Phytocoenotic table of the syntaxa with participation of the Molinia caerulea s.l. in southwestern of Ukraine (clusters 6-9). 